\title{
Flora e estrutura da vegetação secundária após o uso de diferentes trituradores florestais
}

\author{
Mauro Antônio Cavaleiro Macedo Rodrigues ${ }^{(1)}$, Izildinha Souza Miranda( ${ }^{(1)}$ e Maria do Socorro Andrade Kato(2)
}

(1)Universidade Federal Rural da Amazônia, Caixa Postal 917, CEP 66077-530 Belém, Pará. E-mail: mac.macedo@uol.com.br, izildinha.miranda@ufra.edu.br (2)In memoriam.

\begin{abstract}
Resumo - O objetivo deste trabalho foi caracterizar e comparar a estrutura de florestas secundárias, com quatro anos de idade, no nordeste do Pará, que foram formadas após o uso dos trituradores florestais Ahwi FM600 (T1) e Tritucap (T2). O estudo foi realizado em cinco áreas de pequenos produtores, cada uma dividida em parcelas que totalizavam $60 \mathrm{~m}^{2}$ por área de estudo. Foi encontrada diversidade média (H’) de 2,84 para as áreas T1 e 2,95 para as T2. A densidade média foi 530.167 indivíduos ha-1 nas áreas T1 e 459.556 indivíduos ha-1 nas T2; a média de área basal das T1 foi 1,25 $\mathrm{m}^{2} \mathrm{ha}^{-1} \mathrm{e}$ a das T2, 4,55 $\mathrm{m}^{2} \mathrm{ha}^{-1}$; a biomassa seca estimada para as áreas T1 foi de 2,37 $\mathrm{t} \mathrm{ha}^{-1}$ e 6,68 $\mathrm{t} \mathrm{ha}^{-1}$ para as T2. Ocorrem diferenças estruturais nas florestas secundárias, formadas após a utilização dos trituradores.
\end{abstract}

Termos para indexação: Amazônia, agricultura, corte, biomassa.

\section{Flora and structure of the secondary vegetation after the use of different crushers}

\begin{abstract}
The objective of this study was to characterize and compare the structure of secondary forests with four years of age, in the northeast of Pará State, which were formed after use of the crushers Ahwi FM600 (T1) and Tritucap (T2). The study was accomplished in five areas of small producers, each one were divided in plots with $60 \mathrm{~m}^{2}$ for study area. The mean diversity (H') was 2.84 for T1 areas and 2.95 for T2 areas; mean density was 530,167 ind. ha $\mathrm{A}^{-1}$ in $\mathrm{T} 1$ and 459,556 ind. ha ${ }^{-1}$ in T2; mean basal area of T1 was $1.25 \mathrm{~m}^{2}$ ha $^{-1}$ and $4.55 \mathrm{~m}^{2}$ ha-1 for T2; estimate dry biomass for T1 was $2.37 \mathrm{t} \mathrm{ha}^{-1}$ and $6.68 \mathrm{t} \mathrm{ha}^{-1}$ for $\mathrm{T} 2$. There are structural differences on secondary forests formed after the use of both crushers.
\end{abstract}

Index terms: Amazonia, agriculture, slash, biomass.

\section{Introdução}

A agricultura tradicional, com as fases de derruba da floresta, queima, plantio agrícola e pousio (fase de reconstituição florestal) (Chinene \& Dynoodt, 1994), tem sido considerada a perturbação antrópica predominante na bacia amazônica (Kennard, 2002). Este modelo secular de agricultura utiliza o fogo como principal ferramenta de eliminação da cobertura vegetal. A insustentabilidade deste modelo é caracterizada pelo progressivo declínio da produção, pela perda do poder de regeneração e conseqüente empobrecimento da vegetação sucessional, notada pela presença proeminente de vegetação herbácea e pelo retardamento da fase de domínio da vegetação lenhosa.

A agricultura de derruba-queima, em áreas da floresta amazônica, afeta a composição das espécies e conseqüentemente a densidade, estrutura e biomassa das florestas secundárias (Uhl, 1987), mas também é considerada um sistema capaz de manter áreas florestais importantes na paisagem e economia da região (Smith et al., 2003), além de oferecer algumas vantagens que tornam a sua utilização bastante difundida, principalmente o baixo custo, a facilidade de implementação e a necessidade de pouca mão-de-obra (Denich et al., 2004).

Segundo Kato et al. (1999), a melhoria de fertilidade do solo, na agricultura de derruba e queima, depende da quantidade de cinza que provém da biomassa queimada, fato que gera paradoxo, uma vez que esse sistema provoca degradação da vegetação, mediante a eliminação das raízes da vegetação lenhosa, a principal responsável pelo acúmulo de biomassa. A maior parte das árvores, arbustos e espécies de lianas lenhosas, como também herbáceas perenes, possuem regeneração vegetativa por rebrota de raízes ou rizomas que sobrevivem ao ciclo de cultivo (Jacobi, 1997; Denich et al., 2001). 
Denich et al. (2005), em experiência realizada na Amazônia Oriental com manejo de capoeiras livres do fogo, propuseram aos agricultores a utilização de máquinas agrícolas para triturar a capoeira e a colocação do material triturado sobre o solo ("mulch"). O sistema de corte-tritura com o uso do "mulch", possui inúmeros benefícios: conservação dos teores de matéria orgânica e fertilidade, melhoria da estrutura física do solo, manutenção da biota do solo, proteção contra a erosão, manutenção da umidade do solo, contenção dos processos de degradação da biodiversidade, diminuição de plantas daninhas e prolongamento do ciclo de cultivo (Denich et al., 2004, 2005).

A adoção da tecnologia de corte e tritura requer técnicas mais avançadas, pois o corte e a trituração da vegetação lenhosa de forma manual é extenuante, o que dificulta sua utilização pelos produtores (Denich et al., 2004). A concepção de um equipamento para o corte e a trituração da vegetação, além de se adequar ao poder econômico dos produtores, deve, também, visar a conservação dos sistemas radiculares, pois a rebrota de raízes ou rizomas é o principal meio de regeneração vegetativa das espécies de árvores, arbustos e lianas lenhosas, como também herbáceas perenes, que sobrevivem após a perturbação (Denich et al., 2001).

Atualmente dois equipamentos para corte e trituração estão sendo testados, o Tritucap e Ahwi FM600, pois atendem a alguns critérios pré-estabelecidos, tais como: preservação das raízes das espécies lenhosas; realização de corte raso, o que não provoca obstrução das linhas de plantio; execução simultânea das atividades de trituração e deposição do material triturado ("mulch") no solo; são de simples operação e manutenção.

Esses equipamentos possuem características que justificaram a investigação dos efeitos do uso deles na estrutura de florestas secundárias: Awhi FM600 é um equipamento produzido comercialmente, possui inúmeras aplicações, principalmente na construção civil, e seu uso na área florestal é uma adaptação, pesa $2,8 \mathrm{t}$, seu mecanismo de trituração utiliza martelos, que giram em alta freqüência e esmagam a vegetação, necessita passar duas vezes sobre uma mesma área para triturar completamente a biomassa, e tritura material lenhoso de no máximo $30 \mathrm{~cm}$ de diâmetro na superfície do solo, ou vegetação sucessional de até 12 anos de idade; o Tritucap, é um protótipo desenvolvido pela universidade alemã George August Göttingen, exclusivamente para a trituração de florestas secundárias, pesa em torno de
1,3 t, utiliza duas serras circulares e hélices (facas helicoidais) para realizar a tarefa de corte e tritura, necessita passar uma única vez sobre uma mesma área, para triturar completamente a biomassa, e tritura troncos lenhosos de no máximo $10 \mathrm{~cm}$ de diâmetro na superfície do solo, ou florestas secundárias de até quatro anos de idade (Denich et al., 2004).

Este estudo teve como objetivo comparar a flora e a estrutura de florestas secundárias jovens do nordeste paraense, formadas após a utilização de diferentes máquinas trituradoras da biomassa vegetal.

\section{Material e Métodos}

O trabalho foi desenvolvido nos municípios de Igarapé Açu e Marapanim, na região conhecida como Zona Bragantina, nordeste do Estado do Pará, Amazônia Oriental, entre $0^{\circ} 45^{\prime} \mathrm{S}$ e $1^{\circ} 39^{\prime} \mathrm{S}$ e $46^{\circ} 16^{\prime} \mathrm{W}$ e $48^{\circ} 15^{\prime} \mathrm{W}$. $\mathrm{O}$ regime de chuvas da região é 2.200 a $2.800 \mathrm{~mm}$ por ano, e a temperatura média é de $25^{\circ} \mathrm{C}$. O clima é Ami de acordo com o sistema de Köppen. Os solos da região são predominantemente Latossolos de baixa fertilidade e Espodossolos (Moran et al., 2000b).

Foram selecionadas cinco áreas $\left(\mathrm{de} 1.250 \mathrm{~m}^{2}\right) \mathrm{de}$ pequenos produtores, com histórico de uso e idades semelhantes (quatro anos de idade em 2004, momento em que ocorreu a coleta de dados). As áreas tiveram o último ciclo agrícola de um ano e meio (dezembro 1998 a junho 2000), onde foram cultivados: arroz (Oryza sativa L.), milho (Zea mays L.), feijão-caupi (Vigna ungüiculata (L.) Walp.) e mandioca (Manihot esculenta Crantz.). Antes do ciclo agrícola, todas as áreas tiveram suas vegetações trituradas (dezembro1998). Duas das cinco áreas, localizavam-se na comunidade São Matias, pertencente ao Município de Igarapé Açu e tiveram suas vegetações trituradas com o triturador Ahwi FM600 (tratamento T1); as outras três áreas localizavam-se na comunidade São João, Município de Marapanim, e tiveram suas vegetações trituradas com o triturador Tritucap (Tratamento T2).

Em cada área foram alocadas, de forma aleatória, quatro unidades amostrais de $5 \times 3 \mathrm{~m}\left(15 \mathrm{~m}^{2}\right)$, num total de $60 \mathrm{~m}^{2}$ de área amostral. Em cada unidade amostral, todos os indivíduos foram inventariados e identificados por comparação no Herbário Eng. Agrôn. João Murça Pires, da Embrapa Amazônia Oriental.

As espécies foram classificadas quanto ao hábito de vida: árvores, arbustos (inclusive os subarbustos), ervas e cipós. Não houve diferenciação quanto ao estágio de vida da planta. 
As comparações florística e estrutural foram realizadas por meio dos parâmetros: riqueza florístrica (S), densidade (D) e densidade relativa (Dr), segundo Brower et al. (1997); diversidade de Shannon-Weaver (H'), segundo Magurran (1988); estimativa de biomassa seca (BS) por meio da equação alométrica, descrita por Uhl et al. (1988) para florestas secundárias: $(\operatorname{lnBS}=-2,17+(1,02 \cdot \operatorname{lnDAP})+0,39 \cdot \ln \mathrm{H}$, em que: $\ln$ é o logaritmo de base neperiana, BS é a biomassa seca em quilograma, DAP é o diâmetro em centímetros, à altura de 1,3 m da superfície do solo, e H é a altura total em metros). No cálculo da biomassa, foram utilizados os valores de árvores e arbustos com altura maior que 1,5 m; apenas nesses indivíduos foram medidas a altura, o DAP e a área basal (AB). O peso da BS em quilograma foi convertido para toneladas por hectare, tendo-se dividido o valor calculado por 1.000 e depois multiplicado por 166,7.

Para uma confirmação das diferenças florísticas e estruturais, foi realizada uma análise de agrupamento com a utilização da distância euclideana, como medida de semelhança, e os grupos hierárquicos foram obtidos por meio do método de Ward, segundo Ludwig \& Reynolds (1988).

\section{Resultados e Discussão}

Nas duas áreas $\left(120 \mathrm{~m}^{2}\right)$ preparadas com o Ahwi FM600 (T1), foram encontrados 6.362 indivíduos (ind.), pertencentes a 44 famílias, 91 gêneros e 118 espécies; a riqueza média foi de $81 \pm 18$ espécies por área, e a densidade média de 530.167 \pm 123.508 ind. ha $^{-1}$. Nesse tratamento, $62,7 \%$ das espécies amostradas ocorreram em uma única área. A diversidade $\left(\mathrm{H}^{\prime}\right)$ média foi de 2,84, a área basal (AB) média foi de $1,25 \mathrm{~m}^{2} \mathrm{ha}^{-1}$, e a biomassa seca (BS) média foi de 2,37 $\mathrm{t} \mathrm{ha}^{-1}$ (Tabela 1).
Nas três áreas $\left(180 \mathrm{~m}^{2}\right)$ preparadas com o tritucap (T2), foram encontrados 8.272 indivíduos pertencentes a 54 famílias, 111 gêneros e 157 espécies; a riqueza média foi de $87 \pm 13$ espécies por área, e a densidade média foi de $459.556 \pm 52.254$ ind. $\mathrm{ha}^{-1}$. Nesse tratamento, $54,1 \%$ das espécies amostradas ocorreram em uma única área. A diversidade (H') média foi de 2,95, a área basal $(\mathrm{AB})$ média foi de $4,55 \mathrm{~m}^{2} \mathrm{ha}^{-1} \mathrm{e}$ a biomassa seca (BS) média foi de 6,68 $\mathrm{tha}^{-1}$ (Tabela 1).

Houve diferenças de riqueza e densidade por hábito entre os dois tratamentos, a porcentagem de espécies herbáceas foi maior no T1 $(22,9 \%)$ do que no T2 $(15,3 \%)$, e a porcentagem de espécies arbóreas foi maior no T2 (28\%) do que no T1 $(21,2 \%)$; as porcentagens de indivíduos de cipós e árvores foram maiores no T2 (18,7 e 16,4\%, respectivamente) do que no T1 (9,6 e 8,6\%, respectivamente) (Tabela 2).

No T1, 69,6\% dos indivíduos pertenciam às dez espécies mais abundantes, que foram: Scleria pterota $(\mathrm{Dr}=14 \%)$, Myrciaria tenella $(12 \%)$, Spermacoce latifolia (8,6\%), Imperata brasiliensis (8,5\%), Pariana campestris (7\%), Spermacoce verticillata $(6,1 \%)$, Panicum maximum (3,9\%), Panicum pilosum (3,5\%), Davilla rugosa (3\%) e Lacistema pubescens (3\%) (Tabela 3).

No T2, 61,8\% dos indivíduos pertenciam às dez espécies mais abundantes, que foram: Pariana campestris $(\mathrm{Dr}=15,7 \%)$, Myrciaria tenella $(9,5 \%)$, Imperata brasiliensis (9,4\%), Davilla rugosa (5,2\%), Homolepis aturensis (4,7\%), Lacistema pubescens (4,4\%), Myrcia bracteata (3,6\%), Myrcia sylvatica $(3,2 \%)$, Inga heterophylla $(3,1 \%)$ e Scleria pterota (3\%) (Tabela 3).

Das 197 espécies inventariadas nas áreas dos dois tratamentos, 78 espécies (40\%) foram comuns aos tratamentos. O dendrograma formado com as abundâncias das espécies revelou dois grupos distintos:

Tabela 1. Densidade (D), riqueza (S), diversidade (H'), área basal (AB) e biomassa seca (BS) das florestas secundárias do nordeste do Pará, formadas após o uso dos trituradores florestais Triturador Ahwi FM600 (T1) e Tritucap (T2).

\begin{tabular}{|c|c|c|c|c|c|c|c|c|c|c|c|}
\hline \multirow[t]{2}{*}{ Triturador } & \multirow[t]{2}{*}{ Áreas } & \multirow{2}{*}{$\begin{array}{c}\mathrm{D} \\
\text { (ind. por } 60 \mathrm{~m}^{2} \text { ) }\end{array}$} & \multirow{2}{*}{$\begin{array}{c}\text { D } \\
\text { (ind. por ha) }\end{array}$} & \multirow[t]{2}{*}{$\mathrm{S}$} & \multirow[t]{2}{*}{$\mathrm{H}^{\prime}$} & \multicolumn{3}{|c|}{$\mathrm{AB}\left(\mathrm{m}^{2} \mathrm{ha}^{-1}\right)$} & \multicolumn{3}{|c|}{$\mathrm{BS}\left(\mathrm{t} \mathrm{ha} \mathrm{l}^{-1}\right)$} \\
\hline & & & & & & Árvores & Arbustos & Total & Árvores & Arbustos & Total \\
\hline \multirow[t]{3}{*}{$\mathrm{T} 1$} & 1 & 2.657 & 442.833 & 68 & 2,63 & 1,02 & 0,46 & 1,48 & 1,89 & 0,59 & 2,48 \\
\hline & 2 & 3.705 & 617.500 & 94 & 3,06 & 0,39 & 0,63 & 1,02 & 0,65 & 1,60 & 2,25 \\
\hline & Média & 3.181 & 530.167 & 81 & 2,84 & 0,70 & 0,54 & 1,25 & 1,27 & 1,10 & 2,37 \\
\hline \multirow[t]{4}{*}{$\mathrm{T} 2$} & 1 & 2.995 & 499.167 & 99 & 3,23 & 5,10 & 1,21 & 6,30 & 6,63 & 2,51 & 9,14 \\
\hline & 2 & 2.402 & 400.333 & 73 & 2,90 & 3,01 & 0,61 & 3,63 & 4,15 & 1,86 & 6,01 \\
\hline & 3 & 2.875 & 479.167 & 90 & 2,71 & 3,41 & 0,32 & 3,73 & 4,44 & 0,45 & 4,89 \\
\hline & Média & 2.757 & 459.556 & 87 & 2,95 & 3,84 & 0,71 & 4,55 & 5,07 & 1,61 & 6,68 \\
\hline
\end{tabular}


o primeiro grupo, composto pelas espécies presentes nas áreas do T1; e o segundo grupo, formado pelas espécies presentes nas áreas do T2 (Figura 1).

Tabela 2. Abundância e riqueza de espécies, de acordo com o hábito de vida, encontradas em florestas secundárias do nordeste do Pará, após o uso dos trituradores Ahwi FM600 e Tritucap.

\begin{tabular}{|c|c|c|c|c|c|c|c|c|}
\hline \multirow{2}{*}{ Hábito } & \multicolumn{4}{|c|}{ Ahwi FM600 } & \multicolumn{4}{|c|}{ Tritucap } \\
\hline & $\mathrm{S}^{(1)}$ & $\%$ & Ind. $^{(2)}$ & $\%$ & $\mathrm{~S}$ & $\%$ & Ind. & $\%$ \\
\hline in & 29 & 24,6 & 613 & 96 & 14 & 27,4 & & \\
\hline & 3 & 31,4 & 2.2 & 35 & & 3 & & \\
\hline & 27 & 22,9 & 2.922 & 45 & & ,3 & & 3 \\
\hline & 25 & 21,2 & 550 & & 44 & 28,0 & & 16,4 \\
\hline otal & 118 & 100,0 & 6.362 & 100,0 & 157 & 100,0 & 8.272 & 100,0 \\
\hline
\end{tabular}

${ }^{(1)}$ Riqueza florística. ${ }^{(2)}$ Número de indivíduos.
A riqueza florística das áreas estudadas foi baixa, mas foi similar à riqueza encontrada por outros autores que estudaram as florestas secundárias de idade semelhante, no nordeste paraense; no entanto, as densidades encontradas neste trabalho foram maiores, provavelmente em razão das diferenças metodológicas que normalmente ocorrem entre diferentes autores.

Denich (1991) encontrou 81 espécies e uma densidade de 80.240 ind. $\mathrm{ha}^{-1}$, em florestas secundárias de Igarapé Açu, nordeste do Pará, com quatro anos de idade, dentro do sistema de agricultura de derruba e queima, ao considerar apenas indivíduos acima de $30 \mathrm{~cm}$ de altura. Leal (2002) encontrou 74 espécies e densidade de 197.200 ind. ha $^{-1}$, em uma floresta formada após a derruba e queima, e 87 espécies e densidade de 179.300 ind. ha ${ }^{-1}$, em uma

Tabela 3. Densidade total $\left(\mathrm{D}=\mathrm{n} \mathrm{n}^{\mathrm{o}}\right.$ de indivíduos por $\left.60 \mathrm{~m}^{2}\right)$ e relativa $(\mathrm{Dr})$ das 30 espécies mais abundantes, levantadas em florestas secundárias do nordeste do Pará, formadas após o uso dos diferentes trituradores florestais Ahwi FM600 e Tritucap.

\begin{tabular}{|c|c|c|c|c|c|c|c|}
\hline \multirow[t]{2}{*}{ Família } & \multirow[t]{2}{*}{ Espécie } & \multicolumn{2}{|c|}{ Ahwi FM600 } & \multirow[t]{2}{*}{ Família } & \multirow[t]{2}{*}{ Espécie } & \multicolumn{2}{|c|}{ Tritucap } \\
\hline & & $\mathrm{D}$ & $\operatorname{Dr}(\%)$ & & & $\mathrm{D}$ & $\operatorname{Dr}(\%)$ \\
\hline Cyperaceae & Scleria pterota Presl. & 892 & 14,0 & Poaceae & Pariana campestris Aubl. & 1.297 & 15,7 \\
\hline Myrtaceae & Myrciaria tenella (DC) O. Berg & 779 & 12,2 & Myrtaceae & Myrciaria tenella (DC) O. Berg & 784 & 9,5 \\
\hline Rubiaceae & Spermacoce latifolia Aubl. & 544 & 8,6 & Poaceae & Imperata brasiliensis Trin. & 781 & 9,4 \\
\hline Poaceae & Imperata brasiliensis Trin. & 538 & 8,5 & Dilleniaceae & Davilla rugosa Poir. & 429 & 5,2 \\
\hline Poaceae & Pariana campestris Aubl. & 446 & 7,0 & Poaceae & Homolepis aturensis Chase & 389 & 4,7 \\
\hline Rubiaceae & Spermacoce verticillata $\mathrm{L}$. & 390 & 6,1 & Lacistemataceae & Lacistema pubescens Mart. & 363 & 4,4 \\
\hline Poaceae & Panicum maximum Jacq. & 248 & 3,9 & Myrtaceae & Myrcia bracteata (Rich.)DC & 299 & 3,6 \\
\hline Poaceae & Panicum pilosum $\mathrm{Sw}$. & 224 & 3,5 & Myrtaceae & Myrcia sylvatica DC. & 264 & 3,2 \\
\hline Dilleniaceae & Davilla rugosa Poir. & 191 & 3,0 & Mimosaceae & Inga heterophylla Willd. & 253 & 3,1 \\
\hline Lacistemataceae & Lacistema pubescens Mart. & 190 & 3,0 & Cyperaceae & Scleria pterota Presl. & 249 & 3,0 \\
\hline Mimosaceae & Inga heterophylla Willd. & 146 & 2,3 & Capparaceae & Cleome sp. & 229 & 2,8 \\
\hline Poaceae & Paspalum maritimum Trin. & 119 & 1,9 & Connaraceae & Rourea doniana Baker & 155 & 1,9 \\
\hline Myrtaceae & Myrcia sylvatica DC. & 103 & 1,6 & Connaraceae & Rourea ligulata Baker & 123 & 1,5 \\
\hline Lamiaceae & Hyptis atrorubens Poit. & 99 & 1,6 & Myrtaceae & Myrciaria floribunda Berg. & 122 & 1,5 \\
\hline Connaraceae & $\begin{array}{l}\text { Bernardinia fluminensis (Gardner) } \\
\text { Planch. }\end{array}$ & 78 & 1,2 & Sapindaceae & Serjania paucidentata DC. & 103 & 1,2 \\
\hline Myrtaceae & Myrcia bracteata (Rich.) DC. & 76 & 1,2 & Flacourtiaceae & Casearia arborea Urb. & 93 & 1,1 \\
\hline Boraginaceae & Cordia multispicata Cham. & 73 & 1,1 & Connaraceae & Bernardinia fluminensis Planch. & 90 & 1,1 \\
\hline Bignoniaceae & $\begin{array}{l}\text { Memora allamandiflora } \\
\text { Bureau K.Schum. }\end{array}$ & 60 & 0,9 & Bignoniaceae & $\begin{array}{l}\text { Memora allamandiflora Bureau } \\
\text { ex K.Schum. }\end{array}$ & 80 & 1,0 \\
\hline Euphorbiaceae & Phyllanthus orbiculatus Rich. & 59 & 0,9 & Rhamnaceae & Gouania cornifolia Reiss. & 79 & 1,0 \\
\hline Melastomataceae & $\begin{array}{l}\text { Pterolepis trichotoma (Rottb.) } \\
\text { Cogn. }\end{array}$ & 57 & 0,9 & Solanaceae & Solanum caavurana Vell. & 78 & 0,9 \\
\hline Connaraceae & Rourea ligulata Baker & 55 & 0,9 & Annonaceae & Guatteria poeppigiana Mart. & 74 & 0,9 \\
\hline Asteraceae & Wulffia baccata (L.) Kuntze & 46 & 0,7 & Caesalpiniaceae & $\begin{array}{l}\text { Cassia hoffmannseggii Benth. in } \\
\text { Mart. }\end{array}$ & 73 & 0,9 \\
\hline Asteraceae & Bidens cynapiifolia Kunth. & 45 & 0,7 & Asteraceae & Wulffia baccata (L.) Kuntze & 72 & 0,9 \\
\hline Cyperaceae & Dichromena ciliata Vahl & 41 & 0,6 & Apocynaceae & $\begin{array}{l}\text { Tabernaemontana angulata } \\
\text { Mart. ex Müll. Arg. }\end{array}$ & 65 & 0,8 \\
\hline Flacourtiaceae & Banara guianensis Aubl. & 40 & 0,6 & Flacourtiaceae & Casearia decandra Jacq. & 64 & 0,8 \\
\hline Strelitziaceae & Phenakospermum guanense Endl. & 36 & 0,6 & Caesalpiniaceae & $\begin{array}{l}\text { Senna chrysocarpa (Desvaux) } \\
\text { H.S.Irwin \& R.C. Barneby }\end{array}$ & 62 & 0,7 \\
\hline Sapotaceae & Chrysophyllum sp. & 35 & 0,6 & Flacourtiaceae & Banara guianensis Aubl. & 59 & 0,7 \\
\hline Bignoniaceae & Memora flavida Bureau \& K.Schum. & 34 & 0,5 & Cecropiaceae & Cecropia palmata Willd. & 59 & 0,7 \\
\hline Schizaeaceae & Lygodium venustum $\mathrm{Sw}$. & 28 & 0,4 & Rubiaceae & Sabicea aspera Aubl. & 55 & 0,7 \\
\hline \multirow[t]{2}{*}{ Fabaceae } & $\begin{array}{l}\text { Abarema cochleata } \\
\text { (Wild.) Barnaby \& Grimes }\end{array}$ & 26 & 0,4 & Caesalpiniaceae & Bauhinia macrostachya Benth. & 53 & 0,6 \\
\hline & Outras 88 espécies & 664 & 10,4 & & Outras 127 espécies & 1.376 & 16,6 \\
\hline
\end{tabular}


floresta formada após trituração, ambas com três anos de idade, ao considerar indivíduos acima de $10 \mathrm{~cm}$ de altura. Moran et al. (1996) estimou, por meio de imagem por satélite, uma média de 76 espécies e densidade de 117.433 ind. ha ${ }^{-1}$, em florestas secundárias com três anos de regeneração, na Amazônia Oriental.

Os dois tratamentos apresentaram altas densidades, esta é uma característica dos primeiros estágios da sucessão vegetal; no entanto, a densidade média das áreas do T1 foi maior que nas áreas do T2. As áreas do T1 também apresentaram quase 50\% menos árvores e mais arbusto e ervas do que nas áreas do T2. Essas diferenças encontradas entre as áreas do $\mathrm{T} 1 \mathrm{e} \mathrm{T} 2$ podem indicar diferenças no desenvolvimento sucessional das florestas, pois, segundo alguns autores, a densidade e riqueza dos diferentes hábitos podem indicar o grau de maturidade da floresta. Segundo Leal (2002), nas florestas secundárias do nordeste paraense, a densidade cai de 197.200 ind. ha ${ }^{-1}$ em florestas de 3 anos de idade, para 122.600 ind. ha ${ }^{-1}$ em uma floresta secundária de 10 anos de idade, e a porcentagem de espécies arbóreas aumenta de 23,3 para 40\% entre essas mesmas florestas. De acordo com Moran et al. (2000a), em estágios intermediários da sucessão secundária, o ambiente proporciona uma seleção das espécies, e ocorre uma diminuição da abundância das herbáceas e um aumento da participação das árvores. Para Gómez-Pompa \& Vázquez-Yanes (1981), as ervas heliófilas efêmeras apresentam uma colonização adensada apenas nos primeiros estágios da sucessão.

É, também, possível que a forma de trituração do T1 - esmagamento da vegetação - tenha comprometido a

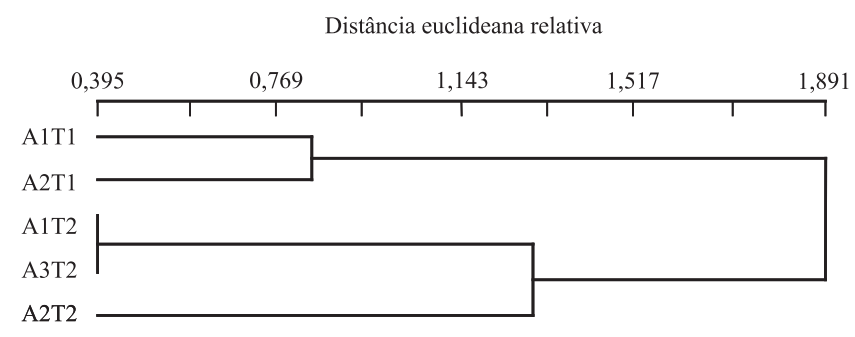

Figura 1. Dendrograma de similaridade, baseado na densidade das 198 espécies pesquisadas em cinco florestas secundárias do nordeste do Pará, formadas após o uso de trituradores, tendo-se usado a distância euclideana relativa, como medida de similaridade, e o método de Ward para agrupamento. A: área amostral; T1: triturador Ahwi FM600; T2: trituradorTritucap. rebrota das árvores, o que resultou em um processo tão danoso para a regeneração quanto o excesso de capina. Áreas em sucessão secundária, que foram capinadas com menos freqüência, tendem a ter maior presença de espécies lenhosas pioneiras, em contraste com o domínio das herbáceas, em áreas que sofreram capina freqüente (Uhl et al., 1987).

Os valores de diversidade encontrados nos tratamentos são baixos, entretanto, são similares aos valores de diversidade encontrados nas florestas secundárias, em estágio inicial de sucessão. Por exemplo, em florestas secundárias de três anos de idade, do nordeste paraense, Moran et al. (1996) estimaram diversidade média de 3,27, e Vieira et al. (2003) estimaram diversidade de 2,54 para árvores com DAP mínimo de $5 \mathrm{~cm}$.

A soma da área basal das espécies no T2 $\left(13,66 \mathrm{~m}^{2} \mathrm{ha}^{-1}\right)$ foi muito superior à do $\mathrm{T} 1\left(2,49 \mathrm{~m}^{2} \mathrm{ha}^{-1}\right)$, o que é indicação do favorecimento do T2 no aumento de diâmetro dos indivíduos, principalmente arbóreos, que, neste tratamento, representou $84,4 \%$ da área basal total, enquanto no $\mathrm{T} 1$ a participação das árvores na área basal total foi de apenas $56,4 \%$. Alguns autores defendem a teoria de que apenas a idade da sucessão florestal não é um parâmetro sustentável para classificar os estágios sucessionais. De acordo com Tucker et al. (1998), o fator principal de diferenciação entre as fases de sucessão é a contribuição das árvores para a área basal total. Segundo Lu et al. (2003), florestas em estágio inicial de sucessão possuem de 0 a $13 \%$ de participação das árvores na área basal total, estágios intermediários de 14 a $49 \%$ e estágios avançados de 50 a $90 \%$. Outros autores consideram apenas a idade para classificar o estágio sucessional das florestas, tais como Saldarriaga et al. (1988) e Uhl et al. (1988), que classificam as florestas secundárias em três fases: inicial ( 0 a 5 anos), intermediária (6 a 15 anos) e avançada (a partir de 15 anos).

Segundo Moran et al. (2000b), florestas tropicais, na fase inicial de sucessão, possuem altura média de $6 \mathrm{~m}$ e áreas basais de menos de $10 \mathrm{~m}^{2} \mathrm{ha}^{-1}$, na fase intermediária possuem altura média de 7 a $15 \mathrm{~m}$ e área basal de 10 a $25 \mathrm{~m}^{2} \mathrm{ha}^{-1}$. Os valores de área basal encontrados no $\mathrm{T} 2$ o aproximam mais do estágio intermediário, apesar de as alturas encontradas serem bem inferiores àquelas descritas pelos autores acima.

A espécie Attalea maripa foi responsável por aproximadamente $40 \%$ da área basal das árvores do estrato superior do T2 e por $24,3 \%$ da área basal total 
desse tratamento, embora essa espécie tenha representado apenas $0,5 \%$ da abundância total da comunidade. Isso possibilita a inferência de que não é a comunidade do T2 que possui uma média de área basal elevada para a sua idade, mas poucos indivíduos destas espécies que elevam a média, uma vez que não ocorreu a espécie Attalea maripa no T1. No entanto, sem a participação dos indivíduos da espécie Attalea maripa do estrato superior, a área basal total média do T2 seria de $6,18 \mathrm{~m}^{2} \mathrm{ha}^{-1}$, ainda muito superior à média de área basal do T1 (2,49 $\left.\mathrm{m}^{2} \mathrm{ha}^{-1}\right)$ (Rodrigues, 2005).

A diferença existente entre os valores de biomassa, apresentados pelos tratamentos, decorreu da diferença de área basal total, altura média e densidade arbórea. Barrios \& Cobo (2004) obtiveram em um sistema de corte e tritura, na Colômbia, uma biomassa de 17,8 t e 6,7 $\mathrm{t} \mathrm{ha}^{-1}$, após 27 meses de sucessão. Moran et al. (2000a) estimaram biomassa seca de $20 \mathrm{t} \mathrm{ha}^{-1}$, em uma floresta secundária de quatro anos na Amazônia Oriental, após o uso de fogo. Assim, os valores de biomassa encontrados neste experimento foram baixos, provavelmente em conseqüência da deficiência de nutrientes do solo, que também limita o crescimento dessas florestas (Zarin et al., 2001). Contudo, as diferenças nas médias de biomassa confirmam a diferença estrutural entre os tratamentos.

O dendrograma de similaridade, formado com a densidade das espécies, revelou uma diferença muito grande entre a florística das florestas formadas nos dois tratamentos. Entre as dez espécies mais abundantes de cada tratamento, seis foram similares; no T1, as espécies Spermacoce latifolia, S. verticillata, Panicum maximum e P. pilosum estavam entre as mais abundantes e representavam $22 \%$ dos indivíduos; no T2, as espéceis Homolepis aturensis, Myrcia bracteata, M. sylvatica e Inga heterophylla, que representavam $15 \%$ dos indivíduos, estavam entre as mais abundantes. Essa diferença, somada ao fato de que apenas $40 \%$ do total de espécies foram comuns aos tratamentos, contribuiu para a formação dos dois grupos.

Entre as dez espécies mais abundantes dos dois tratamentos, também estavam Scleria pterota, Myrciaria tenella, Imperata brasiliensis, Pariana campestris, Davilla rugosa e Lacistema pubescens, que são espécies típicas das florestas secundárias do nordeste paraense (Coelho et al., 2003).

Florestas secundárias de mesma idade podem ter estruturas significantemente diferentes, por causa da influência da fertilidade e estrutura do solo, da precipitação pluvial, histórico de uso da terra, vegetação original, tamanho da área desflorestada e outras atividades humanas (Uhl et al., 1988; Tucker et al., 1998; Moran et al., 2000a, 2000b). Porém, a proximidade geográfica entre as áreas e a semelhança dos históricos de usos indicam que as diferenças aqui encontradas podem ser atribuídas aos diferentes trituradores utilizados.

\section{Conclusões}

1. Ocorre diferença florística e estrutural entre as florestas originadas após o uso da máquina Tritucap (T2) e as florestas originadas após o uso da máquina Ahwi FM600A (T1).

2. O uso da máquina Tritucap resulta em florestas com menor densidade de plantas, porém com maior proporção de árvores e maior área basal e biomassa.

3. O uso da máquina Ahwi FM600 (T1) retarda o crescimento das florestas.

\section{Agradecimentos}

À Capes, pela concessão de bolsa de estudo; à Embrapa Amazônia Oriental, por meio do Projeto Tipitamba, pelo apoio logístico; aos proprietários das áreas estudadas, pela permissão do estudo, ensinamentos e apoio incondicional no período de coleta dos dados; à Danielle Mitja, pelas correções e sugestões.

\section{Referências}

BARRIOS, E.; COBO, J.G. Plant growth, biomass production and nutrient accumulation by slash/mulch agroforestry systems in tropical hillsides of Colombia. Agroforestry Systems, v.60, p.255-265, 2004.

BROWER, J.E.; ZAR, J.H.; ENDE, C.N. von. Field and laboratory methods for general ecology. New York: WCB/McGraw-Hill, 1997. 273p.

CHINENE, V.R.N.; DYNOODT, S. Soil ecology and conservation in the tropics. In: BALAKRISHNAN, M.; BORGSTRÖM, R.; BIE, S.W. (Ed.). Tropical ecosystems: a synthesis of tropical ecology and conservation. Lebanon: International Science Publisher, 1994. p.71-123.

COELHO, R. de F.R.; ZARIN, D.J.; MIRANDA, I.S.; TUCKER, J.M. Análise florística e estrutural de uma floresta em diferentes estágios sucessionais no Município de Castanhal, Pará. Acta Amazonica, v.33, p.563-582, 2003.

DENICH, M. Estudo da importância de uma vegetação secundária nova para o incremento da produtividade do sistema de produção na Amazônia Oriental brasileira. 1991. 283p. Tese (Doutorado) - University of Göttingen, Göttingen. 
DENICH, M.; VIELHAUER, K.; KATO, M. do S.A.; BLOCK, A.; KATO, O.R.; SÁ, T.D. de A.; LÜCKE, W.; VLEK, P.L.G. Mechanized land preparation in forest-based fallow systems: the experience from Eastern Amazonia. Agroforestry Systems, v.6162, p.91-106, 2004.

DENICH, M.; VIELHAUER, K.; SÁ, T.D. de A.; LÜCKE, W.; VLEK, P.L.G. Alternatives to slash-and-burn agriculture: a research approach for the development of a chop-and-mulch system. In: CONFERENCE ON INTERNATIONAL AGRICULTURAL RESEARCH FOR DEVELOPMENT, 1., 2001, Bonn. Proceedings. Bonn: University of Bonn, 2001. p.1-8.

DENICH, M.; VLEK, P.L.G.; SÁ, T.D. de A.; VIELHAUER, K.; LÜCKE, W. A concept for the development of fire-free fallow management in the Eastern Amazon, Brazil. Agriculture, Ecosystems and Environment, v.110, p.43-58, 2005.

GÓMEZ-POMPA, A.; VÁZQUEZ-YANES, C. Successional studies of a rainforest in Mexico. In: WEST, D.C.; SHUGART, H.H.; BOTKIN, D.B. (Ed.). Forest succession: concepts and application. New York: Springer-Verlag, 1981. p.246-266.

JACOBI, I. The contribution from seedlings to the regeneration of the fallow-vegetation in the Eastern Amazon-area. 1997. 148p. Thesis (Ph.D) - University of Hamburg, Hamburg.

KATO, M. do S.A.; KATO, O.R.; DENICH, M.; VLEK, P.L.G. Fire-free alternatives to slash-and-burn for shifting cultivation in the Eastern Amazon region: the role of fertilizers. Field Crops Research, v.62, p.225-237, 1999.

KENNARD, D.K. Secondary forest succession in a tropical dry forest: patterns of development across a 50-year chronosequence in lowland Bolivia. Journal of Tropical Ecology, v.18, p.53-66, 2002.

LEAL, E.C. Potencial de regeneração da capoeira após preparo de área com queima e sem queima na região Bragantina. In: ANGELOMENEZES, M.N.; NEVES, D.P. (Org.). Agricultura familiar: pesquisa, formação e desenvolvimento - interdisciplinaridade para a compreensão da complexidade da agricultura familiar. Belém: Universidade Federal do Pará, Centro Agropecuário, 2002. p.330-400.

LU, D.; MAUSEL, P.; BRONDÍZIO, E.; MORAN, E. Classification of successional forest stages in the Brazilian Amazon basin. Forest Ecology and Management, v.181, p.301-312, 2003.

LUDWIG, J.A.; REYNOLDS, J.F. Statistical ecology: a primer on methods and computing. New York: Wiley, 1988. 337p.

MAGURRAN, A.E. Ecological diversity and its measurement. New Jersey: Princeton University Press, 1988. 179p.

MORAN, E.F.; BRONDÍZIO, E.S.; TUCKER, J.M.; SILVAFORSBERG, M.C. da; FALESI, I.; McCRACKEN, S.D. Strategies for Amazonian forest restoration: evidence for afforestation in five regions of the Brazilian Amazon. In: HALL, A. (Ed.). Amazonia at the crossroads: the challenge of sustainable development. London: Institute for Latin American Studies, University of London, 2000a. p.129-149.

MORAN, E.F.; BRONDÍZIO, E.S.; TUCKER, J.M.; SILVAFORSBERG, M.C. da; McCRACKEN, S.; FALESI, I. Effects of soil fertility and land-use on forest succession in Amazonia. Forest Ecology and Management, v.139, p.93-108, 2000b.

MORAN, E.F.; PACKER, A.; BRONDÍZIO, E.; TUCKER, J.M. Restoration of vegetation cover in the Eastern Amazon. Ecological Economics, v.18, p.41-54, 1996.

RODRIGUES, M.A.C.M. Comparação da estrutura de florestas secundárias formadas a partir de dois diferentes sistemas agrícolas, no nordeste do Estado do Pará, Brasil. 2005. 72p. Dissertação (Mestrado) - Universidade Federal Rural da Amazônia, Belém.

SALDARRIAGA, J.G.; WEST, D.C.; THARP, M.L.; UHL, C. Long-term chronosequence of forest succession in the upper Rio Negro of Colombia and Venezuela. Journal of Ecology, v.76, p.938-958, 1988.

SMITH, J.; FERREIRA, S.; KOP, P.V.D.; FERREIRA, C.P.; SABOGAL, C. The persistence of secondary forests on colonist farms in the Brazilian Amazon. Agroforestry Systems, v.58, p.125135, 2003.

TUCKER, J.M.; BRONDÍZIO, E.S.; MORAN, E.F. Rates of forest regrowth in Eastern Amazonia: a comparison of Altamira and Bragantina regions, Para State, Brazil. Interciencia, v.23, p.64-73, 1998.

UHL, C. Factors controlling succession following slash-and-burn agriculture in Amazonia. Journal of Ecology, v.75, p.377-407, 1987.

UHL, C.; BUSCHBACHER, R.; SERRÃO, E.A.S. Abandoned pastures in Eastern Amazonia. I. Patterns of plant succession. Journal of Ecology, v.76, p.663-681, 1988.

VIEIRA, I.C.G.; ALMEIDA, A.S. de; DAVIDSON, E.A.; STONE, T.A.; CARVALHO, C.J.R. de; GUERRERO, J.B. Classifying successional forests using Landsat spectral properties and ecological characteristics in Eastern Amazônia. Remote Sensing of Environment, v.87, p.470-481, 2003.

ZARIN, D.J.; DUCEY, M.J.; TUCKER, J.M.; SALAS, W.A. Potential biomass accumulation in Amazonian regrowth forests. Ecosystems, v.4, p.658-668, 2001. 